\title{
Transanal Hemorrhoid Dearterialization and mucopexy: a minimal invasive approach for prolapsed hemorrhoids. Four cases report.
}

\author{
Stefan Morarasu ${ }^{*, 1}$, Emmanuel Eguare $^{2}$, Yasir Bashir $^{2}$, Qurat ul Ain $^{2}$, Anca Bradu $^{1}$ \\ 1“Gr. T. Popa” University of Medicine and Pharmacy, lasi, Romania, ${ }^{2}$ Allen Ward, Naas General \\ Hospital, Naas, Co. Kildare, Ireland
}

\begin{abstract}
Hemorrhoidal disease is one of the most frequent causes of anal pathology. Surgical therapy is reserved for patients who do not respond to non-operative management and for advanced third and fourth degree hemorrhoids. Milligan-Morgan and Ferguson hemorrhoidectomies are considered the first-choice therapy, but these procedures are associated with postoperative complications including infection, stenosis, pelvic floor dysfunction, rectal bleeding and postoperative pain. In order to minimize postoperative complications, less invasive procedures have been described and two of them are reported with promising results: Stapled hemorrhoidopexy (PPH) and Transanal Hemorrhoid Dearterialization (THD). THD involves ligation of the hemorrhoidal arteries under Doppler guidance resulting reduced inflow that facilitates reduction in size of hemorrhoids, while suture mucopexy addresses the prolapsed mucosa. THD is proven as a reliable technique for third degree hemorrhoids, but its role in fourth degree hemorrhoids is not generally accepted. Herein we report four cases of prolapsed hemorrhoids which were treated with THD and mucopexy. The procedure was successful in all patients. Photographs taken, before and after the procedure, show an impressive reduction of the prolapsed hemorrhoids. THD and mucopexy appears to be an effective technique with the potential of treating not only third degree, but advanced hemorrhoids as a safe one-day procedure with immediate visible results.
\end{abstract}

Keywords: THD, Transanal hemorrhoidal dearterialization, hemorrhoids, hemorrhoidectomy

\section{Introduction}

Hemorrhoids are cushions of submucosal tissue containing venules, arterioles and smooth-muscle fibers that are normally located in the anal canal and contribute toward maintaining fecal continence and the entire continence mechanism [1]. When hemorrhoids are associated with bleeding, pruritus, mucous discharge per rectum or prolapse, they are referred as symptomatic and in the presence

Received: September 2015; Accepted after review: March 2016; Published: March 2016.

*Corresponding author: Stefan Morarasu, MD, „Gr. T. Popa” University of Medicine and Pharmacy, Iasi, Romania.

Email: morarasu.stefan@gmail.com of symptom associations they are referred to as "hemorrhoidal disease" [2-4]. Even though they are probably one of the most common causes of anal pathology, the true prevalence of hemorrhoids is difficult to establish, because many patients don't seek medical care. Increased abdominal pressure (obesity, pregnancy), constipation and prolonged straining are believed to be the main causes for prolapse of hemorrhoidal tissue [5].

There are mainly three therapy choices for hemorrhoidal disease: (1) medical management and diet, (2) office-based procedures and (3) surgical therapy [6]. Symptomatic first and second degree hemorrhoids are best managed with diet and decrease in straining (high-fiber diet, regular exercise, stool softeners) and with medical 
therapy (topical ointments, creams, suppositories) [7, 8]. Office-based procedures (rubber band ligation, sclerotherapy, infrared photocoagulation, and cryotherapy) are a suitable tool for persistent first and second degree hemorrhoids and for selected third degree cases [1, 2, 8, 9]. Surgical therapy is reserved for patients who fail non-operative management techniques and for advanced prolapsed third and fourth degree hemorrhoids $[10,11]$.

Transanal Hemorrhoid Dearterialization (THD) and mucopexy is the newest addition to a surgeon's repertoire. It was first described by Morinaga et al. [12] and since then the technique and the operative equipment have been improved. THD procedure involves ligation of the hemorrhoidal arteries under Doppler guidance resulting in reduced inflow that facilitates reduction in size of hemorrhoids, while suture mucopexy addresses the prolapsed mucosa [4]. Kjaer et al. [13] reported a low success rate (64\%) after a median follow-up periods of nine months in patients with three and fourth degree hemorrhoids who underwent THD and mucopexy, while other authors described THD as a safe one-day procedure for patients with hemorrhoids [14-16].

Herein we report four cases of advanced prolapsed hemorrhoids, which were treated with THD and mucopexy. We emphasize that THD is an effective procedure with minimal postoperative pain and early return to normal activity.

\section{Cases report}

Four patients (three male, one female) diagnosed with $4^{\text {th }}$ degree hemorrhoids underwent operative management using THD and mucopexy procedure at Naas General Hospital, Naas, Co. Kildare, Ireland. Main complaints were pruritus ani and long standing rectal bleeding. These patients underwent colonoscopy as part of their pre-operative work-up. Preoperatively phosphate enema was prescribed and Digital Rectal Examination was performed to assess any other concomitant anal or/and rectal pathologies.
Procedures were performed under general anesthesia and patients were placed into lithotomy position.

THD device consists of an anoscope equipped on the superior surface with a Doppler sensor and a light source. After digital examination with lubricant, the anoscope was inserted with the Doppler sensor placed at different clock positions $(1,3,5,7,9,11)$ (Figure1) and at each position the Doppler value was noted in order to localize the hemorrhoidal arteries. Once identified, all six arteries were ligated with a 2-0 absorbable suture (Figures 2 and 3 ). The suturing equipment is also specially designed: the needle holder is longer and has a marked line at the tip where the needle should be placed; the needle is $5 / 8$ of a circle, so that it perfectly fits in the suturing groove. When the artery was identified, the needle holder was inserted inside the anoscope with the tip placed into groove at the end of the inside surface of the anoscope and with circular motions of the needle-holder an "8-figure" suture was placed to ligate each artery. All arteries were ligated.

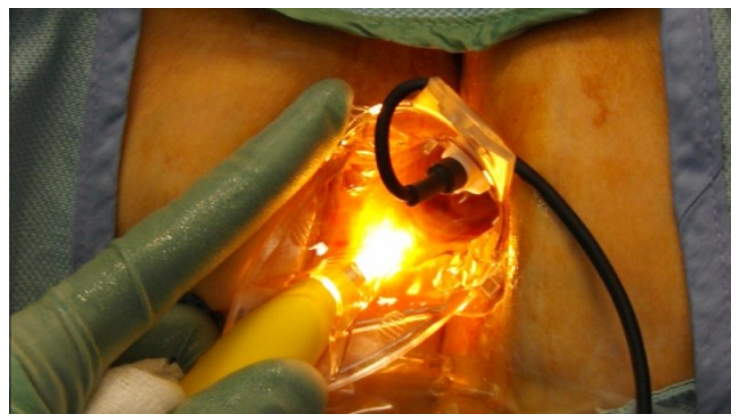

Fig. 1. Anoscope inserted at 1 o'clock in order to assess Doppler signal from the artery.

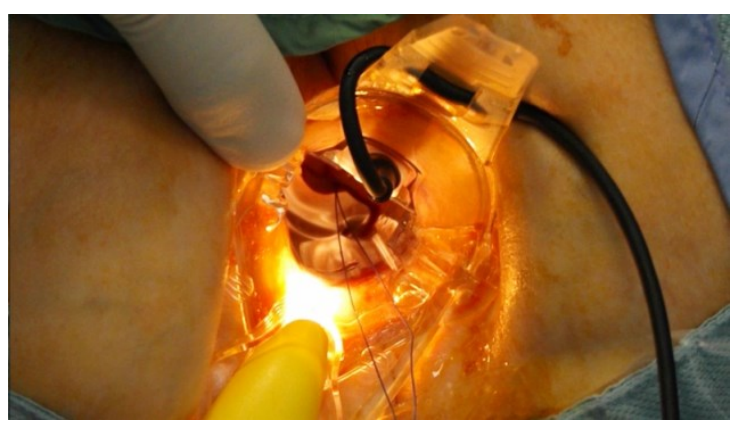

Fig. 2. Hemorrhoidal artery ligated at 1 o'clock position. 


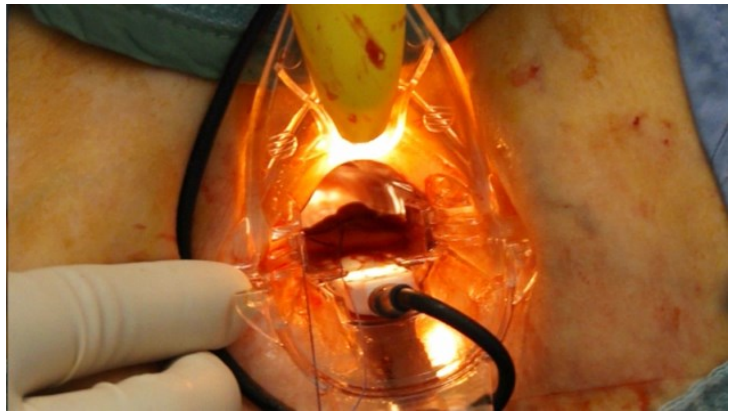

THD procedure ligates the arteries and decreases the arterial blood flow. In order to lift the prolapsed mucosa, mucopexy was necessary. With the anoscope inserted, using the technique described above, multiple running sutures were placed above the dentate line, in order to avoid the anoderm and tied at the proximal end of the hemorrhoid (Figure 4).

Fig. 3. Hemorrhoidal artery ligated at 5 o'clock position.

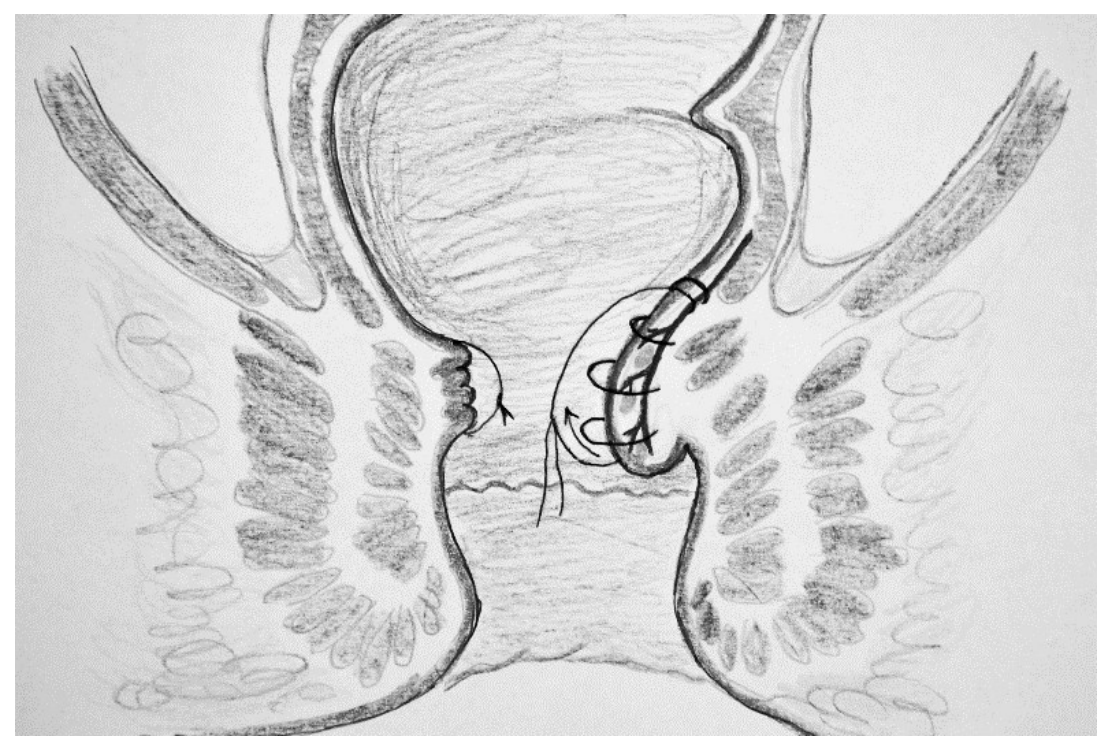

Fig. 4. THD and mucopexy illustration. The hemorrhoidal artery is ligated with an "8 figure" suture and multiple running sutures are used to lift the prolapsed mucosa (left of the image). Notice that all sutures are placed above the dentate line. Once the final knot is tied between the distal and the proximal end, the mucosa is shrunk and fixed, thus the mucopexy is done, as seen on the right of the image.

Anal packing was done using a cylindrical sponge soaked in anesthetic and antiseptic gel (Figure 5).

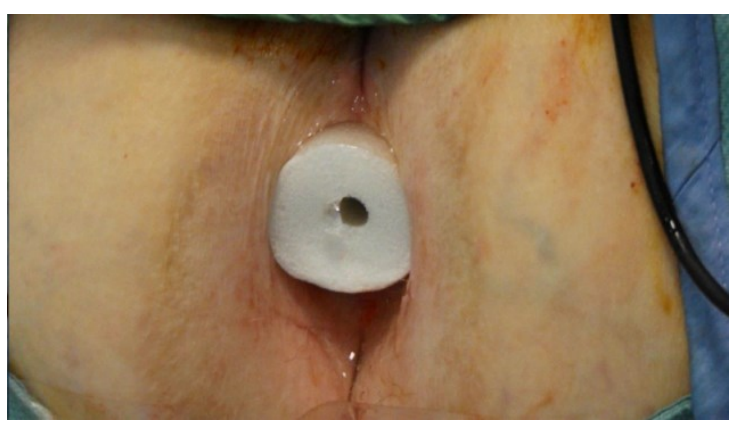

Fig. 5. Anal packing with cylindrical sponge soaked in anesthetic and antiseptic gel.
The mean operative time was approx. 45 min. The procedure was successful in all patients without any intra-operative and postoperative complications. Photographs taken, before and after the procedure, show an impressive reduction of the prolapsed hemorrhoids (Figures 6 - 13).

On ward follow-up patients did not have major symptomatic complaints, except mild nausea, presumably anesthetic related. All patients were discharged in the same day and were prescribed Lactulose $15 \mathrm{mg} /$ twice daily, Flagyl $\circledR$ (metronidazole) 400mg/three times daily, Acetaminophen 1g/three times daily and Difene ${ }^{\circledR}$ (diclofenac) $50 \mathrm{mg} /$ twice daily (each for 5 days). 
Patients were referred back to their general practitioners and were scheduled in

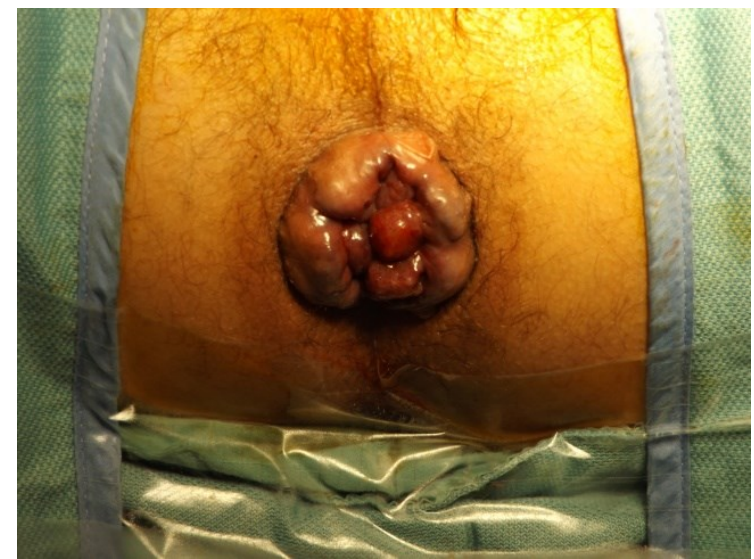

Fig. 6. Patient 1 with prolapsed fourth degree hemorrhoids before the procedure.

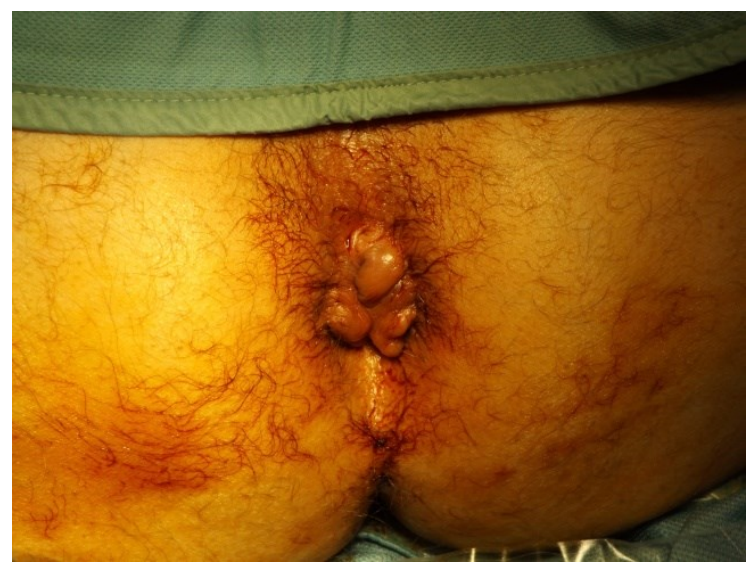

Fig. 8. Patient 2 with fourth degree hemorrhoids before the procedure.

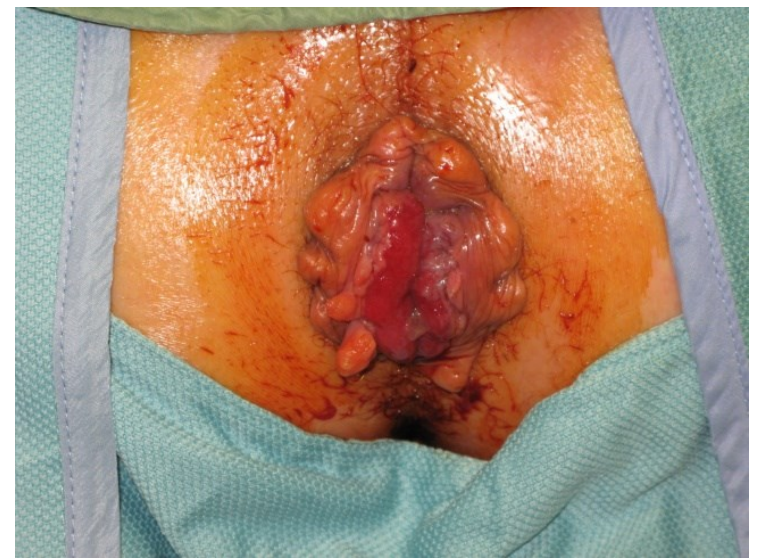

Fig. 10. Patient 3 with advanced fourth degree hemorrhoids before the procedure. Notice the severe mucosal prolapse. the outpatient department for reassessment.

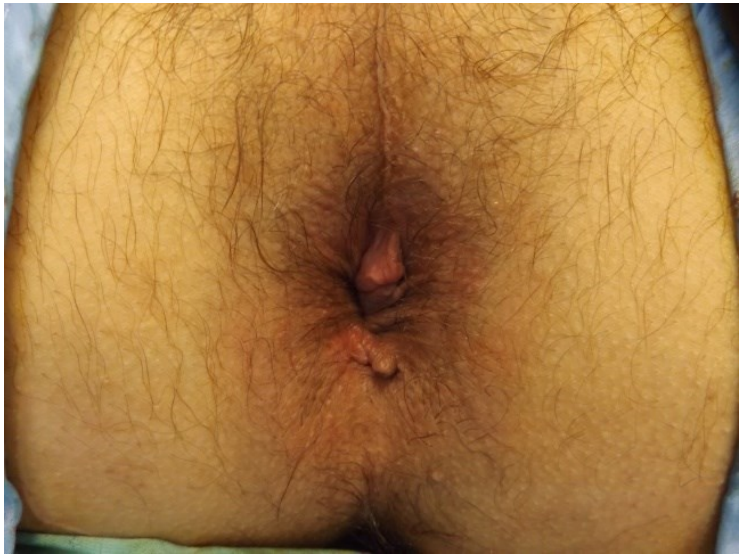

Fig. 7. Patient 1 after the procedure. Prolapsed mucosa is effectively reduced.

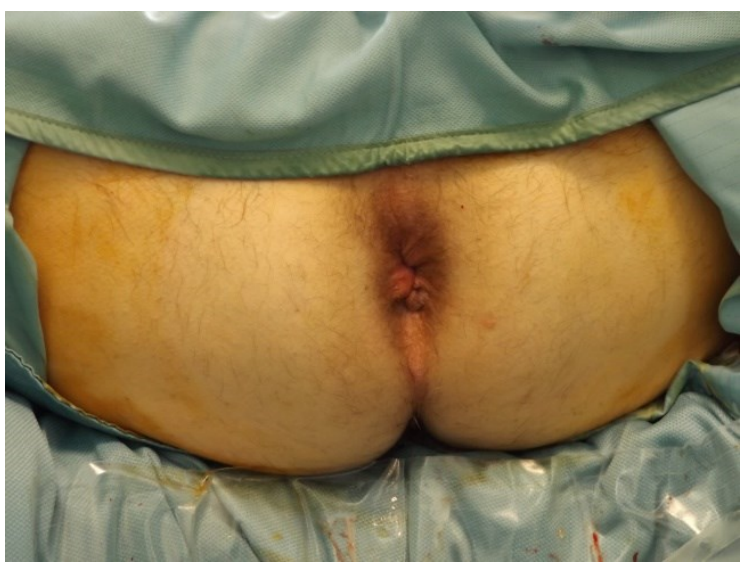

Fig. 9. Patient 2 after the procedure. Hemorrhoids are reduced.

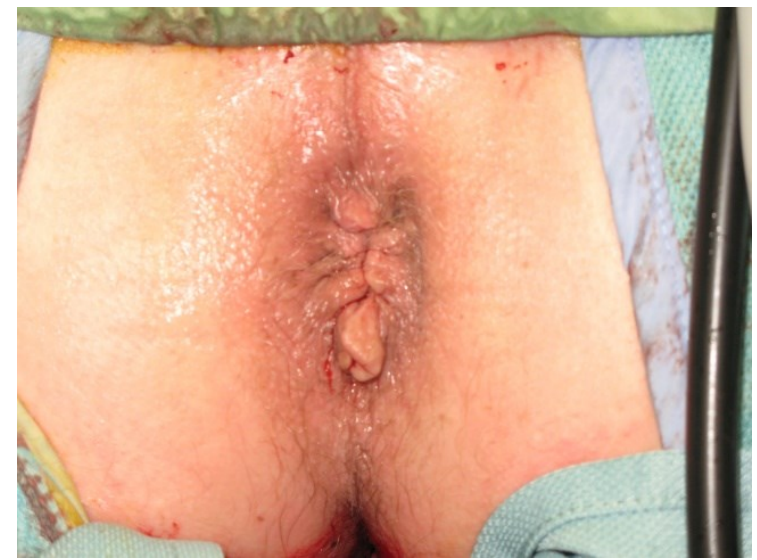

Fig. 11. Patient 3 after the procedure. Prolapsed mucosa is significantly reduced. 


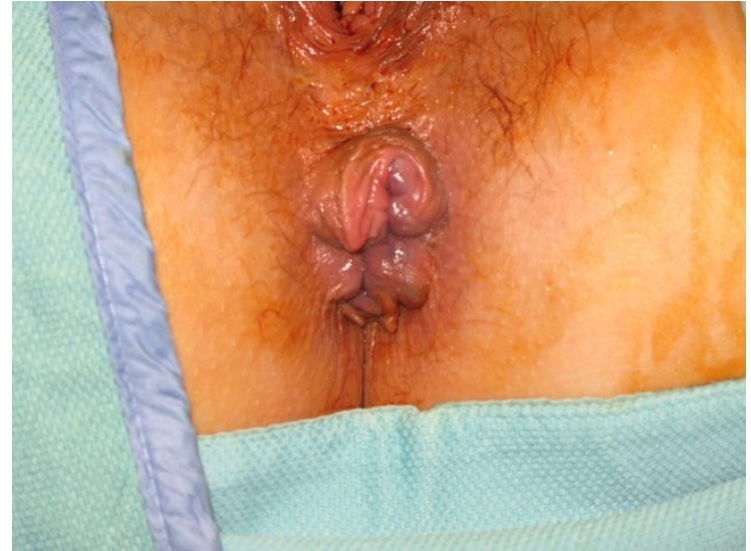

Fig. 12. Patient 4 with fourth degree hemorrhoids before the procedure.

\section{Discussions}

The purpose of this article is to prove the effectiveness of THD in reducing $4^{\text {th }}$ degree prolapsed hemorrhoids as a safe one-day procedure with minimal-to-none pain and other early, hemorrhoidectomy - associated, postoperative symptoms. While the results presented herein prove better outcomes for THD in the first $24 \mathrm{~h}$ post-op, further research is needed to assess the feasibility of this procedure in the long-run. In this case report, there is no data regarding long-term follow up, therefore it is early and beyond this article's reach to say that THD has superior long-term outcomes when compared to other surgical alternatives for $4^{\text {th }}$ degree hemorrhoids. For patients with second and third degree hemorrhoids, Giordano et al. [17] thoroughly evaluated, in a prospective study, the three years outcomes of THD in comparison with stapled hemorrhoidopexy and their results show higher patient satisfaction rates and promising complication rates for THD.

Milligan-Morgan (open) and Ferguson (closed) hemorrhoidectomy techniques are still considered the gold-standard for the operative management of advanced hemorrhoids [4]. However, these procedures are associated with postoperative complications including infection, ano-rectal stenosis, incontinence, urinary retention, severe rectal bleeding and postoperative pain $[4,6,18]$.

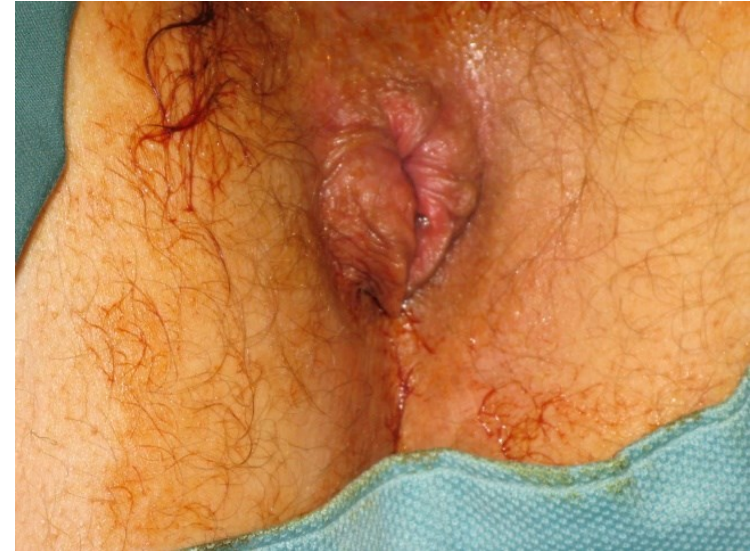

Fig. 13. Patient 4 after the procedure. Hemorrhoidal tissue is effectively reduced.

Firstly described by Milligan and Morgan in 1937 [19], the open hemorrhoidectomy involves a full-thickness excision of the affected mucosa and submucosa: the hemorrhoid is grasped using a forceps and an ellipsoidal incision is made at its base; scissors are used to dissect the remaining hemorrhoid. At the end of the procedure the wound is left open, hence the name "open hemorrhoidectomy". If the mucosa is closed with an absorbable running suture, the procedure is named Ferguson's closed hemorrhoidectomy [10] (Figure 14).

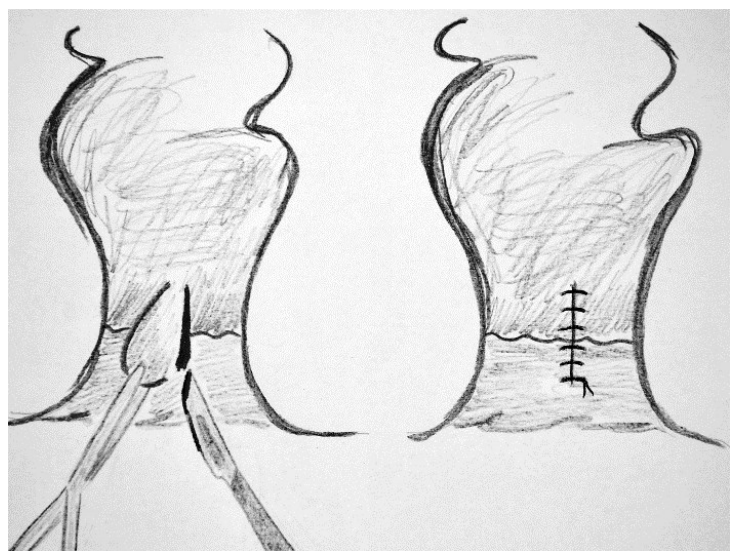

Fig. 14. Ferguson's closed hemorrhoidectomy. The hemorrhoid is grasped using a forceps and incised at its base (right). After the entire hemorrhoid is dissected, the mucosa is closed using an absorbable running suture (left). Note that the incision is made in the anoderm bellow the dentate line, where the skin is highly sensitive to pain. 
In order to minimize complications and postoperative pain, several less invasive procedures have been described and two of them are reported with promising results: $\mathrm{PPH} /$ stapled hemorrhoidopexy and Transanal Hemorrhoid Dearterialization (THD)/Doplerguided hemorrhoidal artery ligation (DGHAL). In the past years, stapled hemorrhoidopexy became less popular even though early postoperative pain is not as severe as after hemorrhoidectomy [20]. Various studies have shown that PPH is associated with a greater recurrence rate [21], higher stenosis rate [15] when compared with the standard hemorrhoidectomy and it is related with other unusual complications (chronic proctalgia, bleeding granulomatous polyps due to retained staples, rectovaginal fistulae etc.) which are not described after Ferguson or Milligan-Morgan approach [18].

Associated with mucopexy, THD appears to be an effective technique for the management of advanced fourth degree hemorrhoids and offers immediate results as seen in the cases presented above. It is a relatively new procedure developed as an alternative to excisional hemorrhoidectomy and it is increasingly being embraced by surgeons for its better outcomes, higher patient satisfaction and fewer postoperative complications [23-26]. For II and III degree hemorrhoids, THD is a widely accepted therapeutic choice, but there is little information of the results of THD for grade IV hemorrhoids [14].

Table. 1. Comparison between open and closed hemorrhoidectomies and THD. THD is associated with a significant shorter hospital stay.

\begin{tabular}{cccc}
\hline & $\begin{array}{c}\text { Open } \\
\text { Hemorrhoidectomy [27] }\end{array}$ & $\begin{array}{c}\text { Closed } \\
\text { hemorrhoidectomy [27] }\end{array}$ & THD and mucopexy \\
\hline Mean operative time & approx. 25 min. & approx. 31 min. & approx. 45 min. \\
Hospital days & $3-5$ days & $2-4$ days & one-day case \\
\hline
\end{tabular}

Milligan-Morgan (open)/Ferguson (closed) hemorrhoidectomy techniques withstood over the years because of their efficacy, but the notorious postoperative pain pushed researchers in developing less invasive treatments. THD is a non-excisional operative technique that spares the anoderm; therefore it is associated with less postoperative pain when compared to the excisional approaches. Popo et al. [28] published a comparison between THD and two excisional procedures: Milligan-Morgan and Whitehead hemorrhoidectomy; they found that THD is linked with shorter hospital stay, less discomfort in postoperative period and an excellent aesthetic effect. Another famous less invasive treatment for hemorrhoidal disease that offers superior results to the classic procedures is stapled hemorrhoidopexy (PPH). Several studies comparing THD and PPH $[29,30]$ described that both procedures seem an effective and safe treatment for hemorrhoids, but THD seems to be superior when it comes to pain score and return to normal daily activities. Verre et al. [31] report better results in terms of pain and postoperative complications after THD surgery, while Lucarelli et al. [32] describe a higher recurrence rate after THD and similar long-term follow-up results regarding symptom control and patient satisfaction.

The approach and equipment used in the cases presented above are relatively new, being described by Ratto in 2014 [33].

\section{Conclusions}

Since its first report, the THD procedure has been constantly modified and improved. THD is a promising minimal invasive technique and it offers major advantages such as higher patient satisfaction, fewer complications, less pain and earlier return to normal daily 
activities. Even so, many surgeons do not embrace this procedure as the standard of care for grade III-IV hemorrhoids mainly because of high recurrence rate, expensive equipment and lack of success in reducing severe prolapsed mucosa. These patients had severe mucosal prolapse and, as seen in pictures, ligation of all arteries accompanied by a continuous suture placed above the dentate line can effectively reduce and fix even prolapsed hemorrhoids. We believe that THD with mucopexy can be safely used in advanced fourth degree hemorrhoids as a safe one-day procedure with immediate visible results. Without proper equipment, finding and ligating the arteries is technically demanding and can lead to poor results. Even though it is expensive, this new THD equipment provides standardization, reduces recurrence rate and it

\section{References}

1. Brunicardi FC, Andersen DK, Billiar TR, et al. Schwartz's Principles of Surgery, 9e: The McGraw-Hill Companies, Inc. 2010.

2. Kaidar-Person $\mathrm{O}$, Person $B$, Wexner SD. Hemorrhoidal disease: a comprehensive review. J Am Coll Surg 2007; 204:102-117.

3. Jacobs D. Hemorrhoids. N Engl J Med 2014; 371:944-951.

4. LaBella GD, Main WPL, Hussain LR. Evaluation of transanal hemorrhoidal dearterialization: a single surgeon experience. Tech Coloproctol 2015; 19:153-157.

5. Lohsiriwat V. Hemorrhoids: From basic pathophysiology to clinical management. World J Gastroenterol 2012; 18(Suppl 17):2009-2017.

6. Beck DE, Roberts PL, Saclarides TJ, et al. The ASCRS Textbook of Colon and Rectal Surgery, second Edition. Springer Science + Business Media, 2011:175-202.

7. MacKay D. Hemorrhoids and varicose veins: a review of treatment options. Altern Med Rev 2001; 6(Suppl 2):126-140.

8. Mounsey AL, Halladay J, Sadiq TS. Hemorrhoids. Am Fam Physician 2011; 84(Suppl 2): 204-210.

9. Brill $A L$, Fleshman JW, Ramshaw BJ, et al. Minimally invasive procedures: What family physicians need to know? J Fam Pract 2005; 54(Suppl 1):1-24.

10. Tsikitis VL. Anal surgery for hemorrhoids [http://emedicine.medscape.com/article/158235 8-overview available at 03.31.2016]. may become cost-effective, because postoperative hospitalization costs are kept at a minimum. Further research is needed to establish which of the two well-known less invasive procedures (PPH and THD) is the treatment of choice for IV degree hemorrhoids.

\section{Acknowledgements}

This article was designed and written with special contributions from Mr. Emmanuel Eguare and his operative team. The cases presented in this manuscript are patients treated by Mr. Emmanuel Eguare at Naas General Hospital and the paper was conducted during my ( $\mathrm{S}$. Morarasu) summer elective clinical clerkship in Mr. Eguare's clinic. The corresponding author would like to express his deepest gratitude to all the staff at Naas General Hospital, Naas, Co. Kildare, Ireland.
11. Cerato MM, Cerato NL, Passos $P$ et al. Surgical treatment of hemorrhoids: a critical appraisal of the current options. $A B C D$ Arq Bras Cir Dig 2014; 27(Suppl 1):66-70.

12. Morinaga $K$, Hasuda $K$, Ikeda $T$. A novel therapy for internal hemorrhoids: ligation of the hemorrhoidal artery with a newly devised instrument (Moricorn) in conjunction with a Doppler flowmeter. Am J Gastroenterol 1995; 90(Suppl 4):610-3.

13. Kjaer S, Lund HK, Schulze S, Bisgaard T. Limited success in patients treated with transanal hemorrhoidal dearterialisation. Dan Med J 2014; 61(Suppl 12):A4971.

14. Giordano P, Tomasi I, Pascariello A, Mills E, Elahi S. Transanal dearterialization with targeted mucopexy is effective for advanced hemorrhoids. Colorectal Disease 2014; 16:373376.

15. Noguerales F, Diaz R, Salim B et al. Treatment of hemorrhoids by transanal hemorrhoidal dearterialisation. Experience of Several Specialized Units. Cir Esp 2015; 93(Suppl 4):236-240.

16. Ratto $C$, Giordano $P$, Donisi $L$ et al. Transanal Hemorrhoid Dearterialization (THD) for selected four-degree hemorrhoids. Tech Coloproctol 2011; 15(Suppl 2):191-197.

17. Giordano P, Nastro P, Davies A, Gravante G. Prospective evaluation of stapled hemorrhoidopexy versus transanal haemorrhoidal dearterialisation for stage II and 
III haemorrhoids: three-year outcomes. Tech Coloproctol 2011; 15(Suppl 1):67-73.

18. Pescatori M. Prevention and Treatment of Complications in Proctological Surgery. Springer - Verlang Italia, 2012.

19. Agbo SP. Surgical Management of Hemorrhoids. J Surg Tech Case Rep. 2011; 3(Suppl 2):68-75.

20. Tjandra JJ, Chan MK. Systematic review on the procedure for prolapse and hemorrhoids (stapled hemorrhoidopexy). Dis Colon Rectum 2007; 50(Suppl 6):878-892.

21. Rahman ASMT, Rahman ASMZ, Biswas SK et al. Stapled hemorrhoidopexy compared with conventional hemorrhoidectomy - A systematic review. Faridpur Med. Coll. J. 2012; 7(Suppl 1):37-41.

22. Senagore $A J$, Singer $M, A b c a r i a n ~ H$ et al. Procedure for Prolapse and Hemorrhoids (PPH) Multicenter Study Group. A prospective, randomized, controlled multicenter trial comparing stapled hemorrhoidopexy and Ferguson hemorrhoidectomy: perioperative and one-year results. Dis Colon Rectum 2004; 47(Suppl 11):1824-1836.

23. Ratto C, Parello A, Veronese E et al. Dopplerguided transanal haemorrhoidal dearterialization for haemorrhoids: results from a multicentre trial. Colorectal Disease 2015; 17:10-19.

24. Loganathan A, Das A, Luck A, Hewett $P$. Transanal haemorrhoidal dearterialization for the treatment of grade III and IV haemorrhoids: a 3-year experience. Anz J Surg 2016; 86(12):59-62.

25. Tempel MB, Pearson EG, Page $M$, et al. Survey of patient satisfaction after Dopplerguided transanal haemorrhoidal dearterialization performed in ambulatory settings. Tech Coloproctol 2014; 18:607-610.

26. Talha S, Burke JP, Waldron D, Coffey JC, Condon E. Early quality of life outcomes following Doppler guided transanal hemorrhoidal dearterialisation: a prospective observational study. Acta Gastroenterol Belg 2013; 76(Suppl 2):231-234.

27. Shaikh AR, Dalwani AG, Soomro N. An evaluation of Milligan-Morgan And Ferguson procedures for haemorrhoidectomy at Liaquat University Hospital Jamshoro, Hyderabad, Pakistan. Pak J Med Sci 2013; 29(Suppl 1):122-127.

28. Popov V, Zhivkov E, Tokov P, et al. Advantages of transanal hemorrhoidal dearterialisation as compared to other surgical techniques for the treatment of hemorrhoidal disease. Khirurgiia 2013; 3:4-7.

29. Infantino A, Altomare DF, Bottini $C$, et al. Prospective randomized multicenter study comparing stapler hemorrhoidopexy with doppler-guided transanal hemorrhoids dearterialization for third degree hemorrhoids. Colorectal Disease 2011; 14(Suppl 2):205-211.

30. Tsang YP, Fok KL, Cheung YS, LI KW, Tang $\mathrm{CN}$. Comparison of transanal haemorrhoidal dearterialisation and stapled hemorrhoidopexy in management of hemorrhoidal disease: a retrospective study and literature review. Tech Coloproctol 2014; 18(Suppl 11):1017-1022.

31. Verre L, Rossi R, Gaggelli I, et al. PPH versus THD: a comparison of two techniques for III and IV degree hemorrhoids. Personal experience. Minerva Chir 2013; 68(Suppl6):543-550.

32. Lucarelli $P$, Picchio $M$, Caporossi $M$, et al. Transanal haemorrhoidal dearterialisation with mucopexy versus stapler haemorrhoidopexy: a randomized trial with long-term follow-up. Ann R Coll Surg Engl 2013; 95(Suppl 4):246-251.

33. Ratto C. THD Doppler procedure for hemorrhoids: the surgical technique. Tech Coloproctol 2014; 18:291-298. 